\title{
Evaluation of Predictive Value of Swede Score
}

\author{
${ }^{1}$ Deepthi Ravindranath, ${ }^{2}$ Sivasundari, ${ }^{3}$ Onimi Syamala
}

\section{ABSTRACT}

Objective: The predictive value of colposcopy is not as reliable as it was presumed. The Reid colposcopic index is a prevalently used scoring system which was devised to standardize the colposcopic assessment and to predict the histology. The Swede score formulated by Strander et al. in 2005 is a simple scoring system which includes five variables also including the size of the lesion. This study aims to validate the accuracy of the Swede score in the prediction of high-grade lesions (CIN-II or higher).

Methods: The evaluation was carried out in the Department of Obstetrics and Gynecology at Sri Ramachandra Medical College between January 2015 and July 2015. Seventy-nine patients who attended the gynecology outpatient department who underwent colposcopy and guided biopsy were included. The participating colposcopists were well trained. Swede score was calculated for each patient after colposcopy and noted. It was then evaluated with the final histopathology after either colposcopic biopsy or excisional cervical biopsy. Since it was not a research study and it was an evaluation, the management of patients was not influenced by the Swede score.

Results: Whenever the final diagnosis of cervical lesions was CIN-II or higher, our study showed that the Swede score had $71 \%$ sensitivity, $95 \%$ specificity, $77 \%$ positive predictive value and $94 \%$ negative predictive value.

Conclusion: The specificity for a score of 8 or more was $95 \%$, therefore higher scores within the system may more accurately predict high-grade lesions. Swede score is a valid simplified scoring system in accurately predicting high-grade lesions.

Keywords: Cin, Colposcopy, Swede score.

How to cite this article: Ravindranath D, Sivasundari, Syamala O. Evaluation of Predictive Value of Swede Score. J South Asian Feder Menopause Soc 2018;6(2):103-105.

Source of support: Nil

Conflict of interest: None

Date of received: 10 January 2018

Date of acceptance: 27 January 2018

Date of publication: July 2018

\footnotetext{
${ }^{1}$ Junior Resident, ${ }^{2,3}$ Associate Professor

${ }^{1-3}$ Department of Obstetrics and Gynecology, Sri Ramachandra Medical College and Research Institute, Sri Ramachandra University, Chennai, Tamil Nadu, India
}

Corresponding Author: Deepthi Ravindranath, Junior Resident, Department of Obstetrics and Gynecology, Sri Ramachandra Medical College and Research Institute, Sri Ramachandra University, Chennai, Tamil Nadu, India, e-mai: deepti.171@gmail.com

\section{INTRODUCTION}

Colposcopy is the standard of care for the evaluation of cervical dysplasias. However, colposcopy being subjective has its limitations due to interobserver variability, and prior information about referral cytology is likely to influence the diagnostic accuracy. ${ }^{1}$ Of late, there has been a disagreement among the colposcopists regarding its accuracy, chiefly with regard to the site requiring biopsy. ${ }^{2}$

Therefore, a standardized and validated scoring system will not only help in accurately finding or excluding high-grade lesions, but it will also systematize observations, and provide a good foundation for colposcopists to evaluate their skill. ${ }^{3}$

The Reid colposcopic index was devised to standardize colposcopic assessment and for the prediction of histopathology. This system used four variables namely degree of acetowhiteness, margins of the lesion, vascular atypia and iodine staining with a total score of 8 and this system was reported to have $97 \%$ accuracy in predicting the most likely histologic findings. ${ }^{4}$

In 2005, Strander et al. designed a simple scoring system, the Swede score". It includes the four variables from the Reid colposcopic index and a 5th variable- - the lesion size along with modifications to the definitions of the scores for the remaining variables.

Bowring et al. in 2010 evaluated the swede score's (Table 1) accuracy of predicting lesions with a final diagnosis of CIN II or higher and their results showed a specificity of $95 \%$ when scoring is $>8$ and also observed no obvious learning curve in using this scoring system between trainees and trained colleagues. ${ }^{5}$

The primary aim of the study was to validate the accuracy of Swede score and help systematize observations among all colposcopists within the Department of Gynecology in SRMC, and thereby improving the chances of preventing cervical cancer.

\section{METHODS}

The study included women who attended the department of an outpatient clinic in Obstetrics and Gynecology at Sri Ramachandra Medical College and Research Institute, Chennai, Tamil Nadu between January 2015 and July 2015. Ethical clearance was obtained from the institute's ethical clearance committee.

A total of 79 patients with indications for colposcopy like abnormal cytology, VIA/VILI positive patients were 


\begin{tabular}{|c|c|c|c|c|}
\hline \multicolumn{5}{|c|}{ Table 1: Swede score ${ }^{5}$} \\
\hline & 0 & 1 & 2 & Score \\
\hline $\begin{array}{l}\text { Acetoup- } \\
\text { take }\end{array}$ & $\begin{array}{l}\text { None or } \\
\text { Transparent }\end{array}$ & $\begin{array}{l}\text { Shady, } \\
\text { milky (not } \\
\text { transparent } \\
\text { not opaque) }\end{array}$ & $\begin{array}{l}\text { Distinct, } \\
\text { opaque white }\end{array}$ & \\
\hline $\begin{array}{l}\text { Margins/ } \\
\text { surface }\end{array}$ & Diffuse & $\begin{array}{l}\text { Sharp but } \\
\text { irregular, } \\
\text { jagged, } \\
\text { "geographical" } \\
\text { satellites }\end{array}$ & $\begin{array}{l}\text { Sharp and } \\
\text { even, the } \\
\text { difference in } \\
\text { surface level, } \\
\text { including } \\
\text { cuffing }\end{array}$ & \\
\hline Vessels & $\begin{array}{l}\text { Fine, } \\
\text { Regular }\end{array}$ & Absent & $\begin{array}{l}\text { Coarse or } \\
\text { stypical }\end{array}$ & \\
\hline $\begin{array}{l}\text { Lesion } \\
\text { size }\end{array}$ & $<5 \mathrm{~mm}$ & $\begin{array}{l}5 \text { to } 15 \mathrm{~mm} \text { or } \\
2 \text { quadrants }\end{array}$ & $\begin{array}{l}>15 \mathrm{~mm} \\
\text { or } 3 \text { to } 4 \\
\text { quadrants or } \\
\text { endocervically } \\
\text { undetermined }\end{array}$ & \\
\hline $\begin{array}{l}\text { lodine } \\
\text { staining } \\
\text { Total } \\
\text { Score }\end{array}$ & Brown & $\begin{array}{l}\text { Faintly or } \\
\text { patchy yellow }\end{array}$ & Distinct yellow & \\
\hline
\end{tabular}

included. All colposcopies were performed by trained personnel (one of the authors).

The Swede score was assigned after performing a colposcopy, and then a colposcopy guided-biopsy was performed. The patient was managed as per standard departmental protocol. Other information that was recorded included age, most recent cytology report, the reason for a referral if any. Women with unsatisfactory colposcopy were excluded from the study group. The histopathological examination was carried out by the institution's pathology laboratory and resulted.

The recorded scores were tabulated and compared with their respective histopathological diagnosis. The specificity, sensitivity, positive and negative predictive value were assessed for each score and the statistical analysis was performed by the statistical package for social sciences (SPSS) version 20 software.

\section{RESULTS}

Seventy-nine women were included in this study all of whom were married and were between the age group of 20 to 65 years. The mean age of the patients was 35 years (24-59 years).

The most common indication for colposcopy was VIA positive $76 \%(60 / 79)$ followed by HSIL in cytology $11.4 \%$ (9/79), VILI positive 6.3\% (5/79), ASCUS in cytology 5.1\% (4/79) and one woman with LSIL is cytology.

Of the 79 women the final histopathology revealed 3 (3.8\%) women with CIN-I, 3 (3.8\%) women with CIN-II, $6(7.6 \%)$ women with CIN-III, 5 (6.3\%) women with invasive carcinoma of cervix and 7 (8.9\%) women with negative for dysplasia/CIN and 55 (69.6\%) women with chronic cervicitis.
Table 2: High-grade lesions ( $\mathrm{CIN} \geq 2$ ) prediction by Swede score

\begin{tabular}{lllll}
\hline Score & Sensitivity $\%$ & Specificity $\%$ & PPV $\%$ & NPV\% \\
\hline$\geq 2$ & 100 & 15 & 18 & 100 \\
$\geq 3$ & 100 & 31 & 18 & 100 \\
$\geq 4$ & 100 & 34 & 25 & 100 \\
$\geq 5$ & 100 & 68 & 40 & 100 \\
$\geq 6$ & 93 & 83 & 54 & 98 \\
$\geq 7$ & 93 & 94 & 77 & 98 \\
$\geq 8$ & 71 & 95 & 77 & 94 \\
$\geq 9$ & 21 & 100 & 100 & 86 \\
10 & 7 & 100 & 100 & 83 \\
\hline NPV, negative predictive value; PPV, positive predictive value
\end{tabular}

Following an analysis of all the data, the specificity, sensitivity, positive and negative predictive value for each score has been tabulated in Table 2. A score of 8 or more has a specificity of $95 \%$ with a sensitivity of $71 \%$ ( $p$ value 0.000). Decreasing the score to 5 or more for predicting CIN-II or higher improved the sensitivity to $100 \%$ at the expense of specificity (68\%). Lower scores showed a negative predictive value of $100 \%$ whereas a score of 9 or higher showed a positive predictive value of $100 \%$.

The specificity and sensitivity of each individual variable scoring 2 points has been depicted in Graphs 1 and 2 which reveals that each individual variable is specific for CIN 2 or more when scoring a maximum 2 points with margins and surface and vessels being a $98.5 \%$ specific individual variables and iodine uptake being the most sensitive $(85.7 \%)$ individual predictor.

\section{DISCUSSION}

The results are comparable to those found by Bowring et al. ${ }^{5}$ and Strander et al. ${ }^{3}$ The specificity when scoring 8 or more was $95 \%$ which equated with $95 \%$ in Bowring et al. and $90 \%$ in Strander et al. ${ }^{3}$ demonstrating that higher scores strongly correlate CIN2 or higher, in the participants of our study.

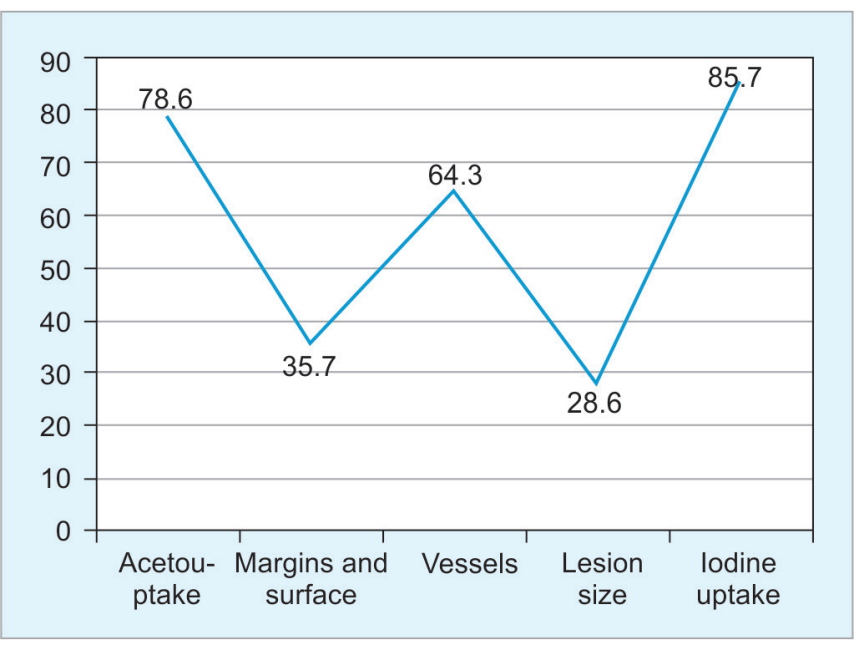

Graph 1: Sensitivity of individual variables scoring 2 points 


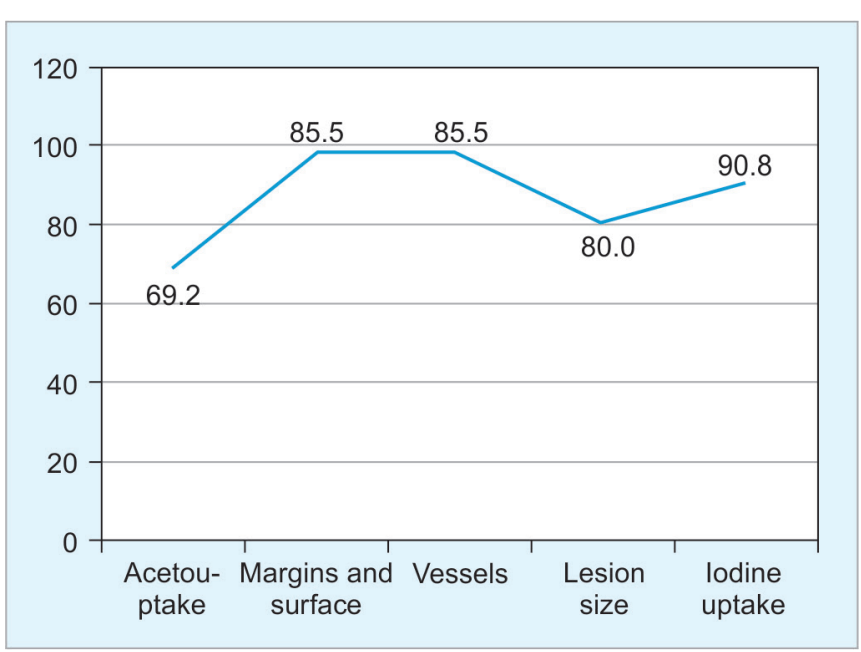

Graph 2: Specificity of individual variables scoring 2 points

The specificity of each individually variable was also comparable to Bowring et al., ${ }^{5}$ and also margins and surface were found to be the most specific individual variable in both the studies.

Verification bias is always a risk in studies involving colposcopy because the outcome is based on biopsy and the site of which is determined by the colposcopic assessment. ${ }^{5}$ Pretorius et al. ${ }^{6}$ showed that even in situations where colposcopy was satisfactory in confirming CIN the ability to detect abnormal areas remained inconsistent. Following biopsies from abnormal and normal regions in colposcopy when their cytology was high-grade, $37.1 \%$ of the CIN 2 or higher lesions were diagnosed from biopsies of 'normal' areas in colposcopy. Although the sensitivity of detecting high-grade lesions does not change significantly by the difference in medical training acquired, the sensitivity is greater when increasing the number of biopsies taken on each patient ${ }^{7}$ which will, however, lead to increased discomfort and morbidity for the patient. ${ }^{8}$ There is also a difference of opinion among colposcopists when reviewing cervical images from women with borderline cytology. ${ }^{9}$

The International Federation for Cervical Pathology and Colposcopy has introduced a set of colposcopy nomenclature in 2002 and had added modifications in 2011 which has facilitated to standardize the way colposcopists construe their examination. ${ }^{10,11}$

The Swede score has helped alleviate many of the insufficiencies of colposcopy and is suggested to be a simple scoring system that can be used by any grade of colposcopists with a small learning curve and has helped in creating a cut off value (scores 8 or higher) at which colposcopy centers may treat patients at first visit without the need for previous histology report. ${ }^{3}$

Swede score has the potential for universal acceptance if further studies are performed by removing the limitations present in the previous studies and if the results are reproducible. In poor-resource settings and third-world countries, this system may have a valuable use in disease prevention.

\section{REFERENCES}

1. Etherington I, Luesley D, et al. Observer variability among colposcopists from the West Midlands region. BJOG: An International Jour-nal of Obstetrics and Gynaecology. 1997;104(12):1380-1384.

2. Jeronimo J, Schiffman M. Colposcopy at a crossroads. American Journal of Obstetrics and Gynecology. 2006;195(2): 349-353.

3. Strander B, Ellstrom-Andersson A, et al. The performance of a new scoring system for colposcopy in detecting high-grade dysplasia in the uterine cervix. Acta Obstetricia et Gynecologica Scandinavica 2005;84(10):1013-1017.

4. Reid R, Scalzi P. Genital warts and cervical cancer. American J Obstetr Gynecol 1985;153(6):611-618.

5. Bowring J, Strander B, et al. The Swede Score. Journal of Lower Genital Tract Dis-ease. 2010;14(4):301-305.

6. Pretorius $\mathrm{R}$, Zhang $\mathrm{W}$, et al. Colposcopically directed biopsy, random cervical biopsy, and endocervical curettage in the diagnosis of cervical intraepithelial neoplasia II or worse. American Journal of Obstetrics and Gynecology. 2004;191(2):430-434.

7. Gage J, Hanson V, et al. Number of Cervical Biopsies and Sensitivity of Colposcopy. Obstetrics and Gynecology. 2006;108(2):264-272.

8. Sharp L, Cotton S, et al. After-effects reported by women following colposcopy, cervical biopsies and LLETZ: results from the TOMBOLA trial. BJOG. 2009;116(11):1506-1514.

9. Massad L, Jeronimo J, et al. Interobserver Agreement in the Assessment of Components of Colposcopic Grading. Obstetrics and Gynecology 2008;111(6):1279-1284.

10. Walker P. International terminology of colposcopy: an updated report from the international federation for cervical pathology and colposcopy. Obstetrics \& Gynecology 2003;101(1): 175-177.

11. Bornstein J, Bentley J, et al. 2011 Colposcopic Terminology of the International Federation for Cervical Pathology and Colposcopy. Obstetrics and Gynecology 2012;120(1): 166-172. 